\title{
VERTEX OPERATOR ALGEBRAS ASSOCIATED TO MODULAR INVARIANT REPRESENTATIONS FOR $A_{1}^{(1)}$
}

\author{
Dražen Adamović And Antun Milas
}

\begin{abstract}
We investigate vertex operator algebras $L(k, 0)$ associated with modular-invariant representations for an affine Lie algebra $A_{1}^{(1)}$, where $\mathrm{k}$ is an'admissible' rational number. We show that VOA $L(k, 0)$ is rational in the category $\mathcal{O}$ and find all irreducible representations in the category of weight modules.
\end{abstract}

\section{Introduction}

Vertex operator algebras (VOA) are mathematical counterparts of conformal field theory (CFT). It is very interesting that some representations of affine Lie algebras carries the structure of VOA (or modules for VOA) [FLM], [FZ], [MP].

The new insight in the theory of representations of VOA was made by Frenkel and Zhu (see [FZ], [Z]) by introducing the associative algebra $A(V)$ associated to VOA $V$. So called $A(V)$-theory gave a theoretically elegant way for the classification of all irreducible representations of $V$ and for calculating the 'fusion rules'. They also introduce the term of rational VOA which is a VOA with a finite number of irreducible modules, such that every finitely generated module is completely reducible.

In $[\mathrm{FZ}],[\mathrm{MP}]$ and $[\mathrm{KWn}]$, the irreducible representations of the VOA $L(k, 0), k \in N$, associated to the irreducible highest weight representations for an affine Lie algebra, were classified. It seems that this case is much simpler because the associative algebra $A(L(k, 0))$ is finite dimensional (see $[\mathrm{KWn}])$.

The main goal of this paper is a classification of the irreducible representations of the simple vertex operator algebra $L(k, 0)$ for $A_{1}^{(1)}$ on the admissible rational level $k$. Our main result is that irreducible $L(k, 0)$ modules from the category $\mathcal{O}$ are exactly modular invariant representations for $A_{1}^{(1)}$. To show this, we use $A(V)$-theory and identify $A(L(k, 0))$ with a certain quotient of $U(g)$. Here we used Malikov-Feigin-Fuchs formula for the singular vectors in the Verma modules. Then, by using classification

Received May 8, 1995.

1991 Mathematics Subject Classification. Primary 17b65; Secondary 17A70, 17B67. 
of the irreducible representations in the category $\mathcal{O}$, we find all irreducible representations in the category of weight modules for $A_{1}^{(1)}$.

Feigin and Malikov in $[\mathrm{FM}]$ have a geometrical approach to a similar problem (see also $[\mathrm{AY}]$ ). They calculated conformal blocks for three admissible modules associated to three different points on $C P^{1}$. We interpret our result of the classification of irreducible modules in terms of conformal blocks considered in $[\mathrm{FM}]$.

\section{Preliminaries}

\subsection{Vertex operator algebras and modules.}

Definition 2.1.1. A vertex operator algebra is a $Z$-graded vector space $V=\bigoplus_{n \in Z} V_{n}$ with a sequence of linear operators $\{a(n) \mid n \in Z\} \subset$ End $V$ associated to every $a \in V$, such that for fixed $a, b \in V, a(n) b=0$ for $n$ sufficiently large. We call a family of generating series

$$
Y(a, z)=\sum_{n \in Z} a(n) z^{-n-1} \in(\text { End } V)\left[\left[z, z^{-1}\right]\right],
$$

vertex operators associated to $a$, if they satisfy the following axioms:

(V1) $Y(a, z)=0$ iff $a=0$.

(V2) There is a vacuum vector, which we denote by 1 , such that

$$
Y(1, z)=I_{V}\left(I_{V} \text { is the identity of End } V\right) .
$$

(V3) There is a special element $\omega \in V$ (called the Virasoro element), whose vertex operator we write in the form

$$
Y(\omega, z)=\sum_{n \in Z} \omega(n) z^{-n-1}=\sum_{n \in Z} L_{n} z^{-n-2}
$$

such that

$$
\begin{gathered}
\left.L_{0}\right|_{V_{n}}=\left.n I\right|_{V_{n}}, \\
Y\left(L_{-1} a, z\right)=\frac{d}{d z} Y(a, z) \text { for every } a \in V, \\
{\left[L_{m}, L_{n}\right]=(m-n) L_{m+n}+\delta_{m+n, 0} \frac{m^{3}-m}{12} c,}
\end{gathered}
$$

where $c$ is some constant in $C$, which is called the rank of $V$.

(V4) The Jacobi identity holds, i.e., 
(3) $z_{0}^{-1} \delta\left(\frac{z_{1}-z_{2}}{z_{0}}\right) Y\left(a, z_{1}\right) Y\left(b, z_{2}\right)$

$$
\begin{aligned}
-z_{0}^{-1} \delta\left(\frac{-z_{2}+z_{1}}{z_{0}}\right) & Y\left(b, z_{2}\right) Y\left(a, z_{1}\right) \\
= & z_{2}^{-1} \delta\left(\frac{z_{1}-z_{0}}{z_{2}}\right) Y\left(Y\left(a, z_{0}\right) b, z_{2}\right)
\end{aligned}
$$

for any $a, b \in V$.

The subspace $I$ of $V$ is called an ideal if $Y(a, z) b \in I\left[\left[z, z^{-1}\right]\right]$ for every $a \in V, b \in I$. Given an ideal $I$ in $V$ such that $1 \notin I, \omega \notin I$, the quotient $V / I$ admits a natural VOA structure (see [FZ]).

Definition 2.1.2. Given a $V O A V$, a representation of $V$ (or $V$-module) is a $Z_{+}$-graded vector space $M=\bigoplus_{n \in Z_{+}} M_{n}$ and a linear map

$$
\begin{gathered}
V \longrightarrow(\text { End } M)\left[\left[z, z^{-1}\right]\right], \\
a \longmapsto Y_{M}(a, z)=\sum_{n \in Z} a(n) z^{-n-1},
\end{gathered}
$$

satisfying

(M1) $a(n) M_{m} \subset M_{m+\operatorname{deg} a-n-1}$ for every homogeneous element a.

(M2) $Y_{M}(1, z)=I_{M}$, and setting $Y_{M}(\omega, z)=\sum_{n \in Z} L_{n} z^{-n-2}$, we have

$$
\begin{aligned}
& {\left[L_{m}, L_{n}\right]=(m-n) L_{m+n}+\delta_{m+n, 0} \frac{m^{3}-m}{12} c,} \\
& Y_{M}\left(L_{-1} a, z\right)=\frac{d}{d z} Y_{M}(a, z)
\end{aligned}
$$

for every $a \in V$.

(M3) The Jacobi identity holds, i.e.,

$$
\begin{aligned}
& z_{0}^{-1} \delta\left(\frac{z_{1}-z_{2}}{z_{0}}\right) Y_{M}\left(a, z_{1}\right) Y_{M}\left(b, z_{2}\right) \\
& -z_{0}^{-1} \delta\left(\frac{-z_{2}+z_{1}}{z_{0}}\right) Y_{M}\left(b, z_{2}\right) Y_{M}\left(a, z_{1}\right) \\
& \quad=z_{2}^{-1} \delta\left(\frac{z_{1}-z_{0}}{z_{2}}\right) Y_{M}\left(Y\left(a, z_{0}\right) b, z_{2}\right)
\end{aligned}
$$

for any $a, b \in V$.

The submodules, quotient modules, irreducible modules and completely reducible modules are defined in the usual way ([FHL]). 
2.2. Associative algebra $A(V)$. Let $V$ be a VOA. For any homogeneous element $a \in V$ and for any $b \in V$, following [Z], we define

$$
a * b=\operatorname{Res}_{z} \frac{(1+z)^{w t a}}{z} Y(a, z) b .
$$

Then extend this product bilinearly to the whole space $V$. Let $O(V)$ be the subspace of $V$ linearly spanned by the elements of type

(6) $\operatorname{Res}_{z} \frac{(1+z)^{w t a}}{z^{2}} Y(a, z) b$ for homogeneous elements $a, b \in V$.

Set $A(V)=V / O(V)$. The multiplication $*$ induces the multiplication on $A(V)$ and $A(V)$ becomes an associative algebra. The image of 1 in $A(V)$ becomes the identity element until the image of $\omega$ is in the center of $A(V)$ (see [Z]). Let $M=\oplus_{n \in Z_{+}} M_{n}$ be a $V$-module. For a homogeneous element $a \in V$, define $o(a)=a(\operatorname{deg} a-1)$. From the definition of $M$, it follows that operator $o(a)$ preserves the grading of $M$.

\section{Theorem 2.2.1.}

(a) On $\operatorname{End}\left(M_{0}\right)$ we have

$$
\begin{aligned}
& o(a) o(b)=o(a * b) \\
& o(x)=0
\end{aligned}
$$

for every $a, b \in V, x \in O(V)$. The top level $M_{0}$ is an $A(V)$ module.

(b) Let $U$ be an $A(V)$-module; there exists a $V$-module $M$ such that the $A(V)$-modules $M_{0}$ and $U$ are isomorphic.

Thus, we have one-to-one correspondence between irreducible $V$-modules and irreducible $A(V)$-modules.

We have the following consequence of the definition of $A(V)$.

Proposition 2.2.2. Let $I$ be an ideal of $V$. Assume $1 \notin I, \omega \notin I$. Then the associative algebra $A(V / I)$ is isomorphic to $A(V) /[I]$, where $[I]$ is the image of $I$ in $A(V)$.

2.3. Vertex operator algebras associated to affine Lie algebras. Let $g$ be a finite-dimensional simple Lie algebra over $C$. The affine Lie algebra $\hat{g}$ associated with $g$ is defined as $g \otimes C\left[t, t^{-1}\right] \oplus C c$ with the usual commutation relations. Let $g=n_{-}+h+n_{+}$and $\hat{g}=\hat{n}_{-}+\hat{h}+\hat{n}_{+}$be the usual triangular decompositions for $g$ and $\hat{g}$ and $P=C[t] \otimes g \oplus C c$ be upper parabolic subalgebra. Let $U$ be any $g$-module. Considering $U$ as a $P$-module, we have the induced module (the generalized Verma module) 
$M(\ell, U)=U(\hat{g}) \otimes_{U(P)} U$, where the central element $c$ acts as multiplication with $l \in C$.

For $\lambda \in h^{*}$, denote the Verma module by $M(\lambda) j$ and its irreducible quotient by $V(\lambda)$.

Set $M(\ell, \lambda)=M(\ell, V(\lambda))$. Let $L(l, \lambda)$ denote its irreducible quotient.

Theorem 2.3.1. ([FZ]) Every $M(\ell, 0), \ell \neq-g$ (where $g$ denotes the dual Coxeter number) has the structure of VOA. Let $U$ be any $g$-module. Then every $M(\ell, U)$ is a module for $M(\ell, 0)$. In particular, $M(\ell, \lambda)$ is a $M(\ell, 0)$ module.

Theorem 2.3.2. The associative algebra $A(M(\ell, 0))$ is canonically isomorphic to $U(g)$ and the isomorphism $F: A(M(\ell, 0)) \rightarrow U(g)$ is given by

$$
F\left(a_{1}\left(-i_{1}-1\right) \cdots a_{n}\left(-i_{n}-1\right) 1\right)=(-1)^{i_{1}+\cdots i_{n}} a_{n} \cdots a_{1} .
$$

for every $a_{1}, \cdots, a_{n} \in g$ and every $i_{1}, \cdots, i_{n} \in Z_{+}$.

\section{Irreducible modules for VOA $L(k, 0)$ in the category $\mathcal{O}$}

3.1. Modular invariant representations for $A_{1}^{(1)}$. Let $g=\operatorname{sl}(2, C)$ with generators $e, f, h$ and relations $[h, f]=-2 f,[h, e]=2 e,[e, f]=h$. Let $\Lambda_{0}, \Lambda_{1}$ denote the fundamental weights for $\hat{g}$, and $\omega$ the fundamental weight for $g$.

Definition 3.1.1. $k=p / q \in Q$ is admissible if $q \in N, p \in Z,(p, q)=1$ and $2 q+p-2 \geq 0$.

In $[\mathrm{KW}], \mathrm{V}$. Kac and M. Wakimoto define modular invariant representations. They also define weights which have admissible level and satisfy some technical conditions (for the definition, see $[\mathrm{KW}]$ ). They call them admissible weights.

The following proposition describes the admissible weights and modular invariant representations on level $k$ :

Proposition 3.1.2. Let $k=p / q \in Q$ be admissible. Set $t=k+2$. Define $P^{k}=\left\{(k-n+m t) \Lambda_{0}+(n-m t) \Lambda_{1}, m, n \in Z_{+}, n \leq 2 q+p-2, m \leq q-1\right\}$.

Let $M$ be any irreducible highest weight module with highest weight $\lambda$. The following statements are equivalent:

(1) $M$ is a modular-invariant.

(2) $\lambda$ is an admissible weight.

(3) $\lambda \in P^{k}$. 
(For proof, see $[\mathrm{KW}]$ ).

We need the following description of the set $P^{k}$ :

Lemma 3.1.3. Let $\lambda \in \hat{h}^{*}$. Then $\lambda \in P^{k}$ if and only if

$$
\langle\lambda, c\rangle=k, \quad\langle\lambda, h\rangle=(N-i t-j)
$$

where $i \in\{0, \cdots, l\}, j \in\{1, \ldots, N\}, N=2 q+p-1, l=q-1$.

By using Corollary 2.1 in $[\mathrm{KW}]$ or the Kac determinant formula, we have

Theorem 3.1.4. Let $k=p / q \in Q$ be admissible. Then

$$
L(k, 0)=M(k, 0) / U(\hat{g}) v_{\text {sing }},
$$

where vector $v_{\text {sing }}$ is the unique singular vector of the weight $k \Lambda_{0}-q(2 q+$ $p-1) \delta+(2 q+p-1) \alpha$.

We also need the following theorem:

Theorem 3.1.5. (Kac-Wakimoto) Let $M$ be a $\hat{g}$-module from the category $\mathcal{O}$ such that for any irreducible subquotient $L(\mu)$ the weight $\mu$ is admissible. Then $\hat{g}$-modul $M$ is completely reducible.

3.2. Malikov-Feigin-Fuchs formula. Recall the Malikov-Feigin-Fuchs result giving the singular vector in form with 'rational powers' (see $[\mathrm{MFF}]$ ).

Theorem 3.2.1. (Malikov-Feigin-Fuchs) The singular vector

$$
v_{\text {sing }}=F(k) .1
$$

generates the maximal submodule of $M(k, 0)$, where

$$
F(k)=e(-1)^{N+l t} f(0)^{N+(l-1) t} \cdots f(0)^{N-(l-1) t} e(-1)^{N-l t},
$$

for $N=2 q+p-1, l=q-1$ and $t=p / q+2$.

Remark 3.2.2. In $[\mathrm{MMF}]$, it was proved that this formula really make sense, because only with commutativity can we transform formula (8) to the usual form in $U(\hat{g})$. 
3.3. Fundamental lemma. First we define

$$
\begin{array}{ll}
\epsilon: \quad & U\left(\hat{n}_{-}\right) \rightarrow U(g) \\
& a_{1}\left(-i_{1}\right) \cdots a_{s}\left(-i_{s}\right) \mapsto a_{1} \cdots a_{s},
\end{array}
$$

for every $a_{1}, \ldots, a_{s} \in g, s \in N$.

In the same way as in $[\mathrm{F}]$, we have

Proposition 3.3.1.

$$
\epsilon(F(k))=\prod_{i=1}^{l} \prod_{j=1}^{N} p_{i, j}(h) e^{N},
$$

where $p_{i, j}(h)=e f+(i t+j-1) h-(i t+j)(i t+j-1)$.

We define the $Z$-grading on $U(\hat{g})$ with

$$
\operatorname{deg} a_{1}\left(-i_{1}\right) \cdots a_{k}\left(-i_{k}\right)=i_{1}+\cdots+i_{k},
$$

for every $a_{1}, \ldots, a_{k} \in g$.

In the following lemma, we use ordinary transposing ${ }^{T}$ in $U(g)$ (see [Dix]).

Lemma 3.3.2. Let $g \in U\left(\hat{n}_{-}\right)$, such that $\operatorname{deg} g=n$. Then we have

$$
\epsilon(g) \equiv(-1)^{n}(F[g .1])^{T} \bmod U(g) n_{-} .
$$

Proof. First notice that $n_{-} .1=0$. Since $\operatorname{deg} g=n$, one can write $g$ in a form

$$
g=\sum_{i=0}^{r} g_{i} f(0)^{i}
$$

where

$$
g_{i}=\sum a_{i_{1}}^{(i)}\left(-j_{1}-1\right) \cdots a_{i_{t}}^{(i)}\left(-j_{t}-1\right),
$$

$a_{i_{1}}^{(i)}, \ldots, a_{i_{t}}^{(i)} \in g, j_{1}, \ldots, j_{t} \in Z_{+}, j_{1}+\cdots+j_{t}+t=n, r \in Z_{+}$, and get

$$
\epsilon(g) \equiv g_{0} \bmod U(g) n_{-} .
$$

Set $a_{i_{j}}=a_{i_{j}}^{(0)}$. Since

$$
g .1=g_{0} .1=\sum a_{i_{1}}\left(-j_{1}-1\right) \cdots a_{i_{t}}\left(-j_{t}-1\right),
$$

we have that

$$
\begin{aligned}
F([g .1])^{T} & =\sum(-1)^{n-t}\left(a_{i_{t}} \cdots a_{i_{1}}\right)^{T} \\
& =(-1)^{n} \sum\left(a_{i_{1}} \cdots a_{i_{t}}\right)
\end{aligned}
$$

and the lemma holds. 

have

Set $Q=F\left(\left[v_{\text {sing }}\right]\right) \in U(g)$. From proposition 3.3.1 and lemma 3.3.2 we

Lemma 3.3.3.

$$
Q^{T} \equiv(-1)^{q(2 q-p-1)} \prod_{i=1}^{l} \prod_{j=1}^{N} p_{i, j}(h) e^{N} \bmod U(g) n_{-}
$$

where polynomials $p_{i, j}$ are as in proposition 3.3.1 .

3.4. Classification of representation. $M(k, 0)$, the vertex operator algebra, has maximal ideal $M^{1}(k, 0)$. It is generated by the vector $v_{\text {sing }}$. Let $L(k, 0)$ be the quotient VOA. Proposition 2.2.2 and Theorem 2.3.2 imply

Proposition 3.4.1. $A(L(k, 0))$ is isomorphic to $U(g) / I$ where $I$ is a twosided ideal generated by the vector $Q$.

Let $U$ be any $A(L(k, 0))$-module. Then $U$ is a $g$-module. We have

Proposition 3.4.2. Let $U$ be any $U(g)$-module. Then the following statements are equivalent:

(1) $U$ is a $A(L(k, 0))$-module,

(2) $Q . U=0$.

Set $R=U(g) \cdot Q$ and $R^{T}=U(g) \cdot Q^{T}$. Clearly $R$ and $R^{T}$ are irreducible $g$-modules and $R \cong R^{T} \cong V(2 N \omega) \cong V^{*}(2 N \omega)$.

From these facts and proposition 3.4.2, one can obtain

Lemma 3.4.3. Let $V(\mu)$ be the irreducible highest weight g-module with the highest weight vector $v_{\mu}$. The following statements are equivalent:

(i) $V(\mu)$ is a $A(L(k, 0))$-module,

(ii) $R V(\mu)=R^{T} V(\mu)^{*}=0$,

(iii) $R_{0} v_{\mu}=R_{0}^{T} v_{\mu}^{*}=0$,

where $R_{0}\left(R_{0}^{T}\right)$ denotes the zero-weight subspace of $R\left(R^{T}\right)$.

For $p \in S(h)$ and $\mu \in h^{*}$, define $p(\mu) \in C$ with $p(h) . v_{\mu}=p(\mu) v_{\mu}$.

Let $u_{1} \in R_{0}$ and $u_{2} \in R_{0}^{T}$. Clearly there exists unique polynomials $p_{1}, p_{2} \in S(h)$ such that

$$
u_{1} \equiv p_{1}(h) \bmod U(g) n_{+} \quad u_{2} \equiv p_{2}(h) \bmod U(g) n_{-} .
$$

Then $u_{1} \cdot v_{\mu}=p_{1}(\mu) v_{\mu}$ and $u_{2} \cdot v_{\mu}^{*}=p_{2}(-\mu) v_{\mu}^{*}$.

We have

Lemma 3.4.4. There is a one-to-one correspondence between each two of the following three sets:

(1) $\mu \in h^{*}$ such that $V(\mu)$ is $A(L(k, 0))$-module, 
(2) $\mu \in h^{*}$ such that $p_{1}(\mu)=0$,

(3) $\mu \in h^{*}$ such that $p_{2}(-\mu)=0$.

3.5. The main theorem. The following lemma is obtained by direct calculation:

\section{Lemma 3.5.1.}

$\left[f^{N}, e f+(i t+j-1)(h-(i t+j))\right]=(-N-1+i t+j)(h-i t-j+N) f^{N}$

Proposition 3.5.2. All irreducible $A(L(k, 0))$-modules from the category $\mathcal{O}$ are $V(r \omega), r \in S$, where

$$
S=\{N-i t-j: i=0, \ldots, l ; j=1, \ldots, N\} .
$$

Proof. Let $u \in R_{0}^{T}$. Then $u=(\text { ad } f)^{N} \cdot Q^{T} \equiv f^{N} Q^{T} \bmod U(g) n_{-}$. By using lemma 3.5.1 we have

$$
u \equiv c_{1} \prod_{i=1}^{l} \prod_{j=1}^{N} q_{i, j}(h) f^{N} e^{N} \bmod U(g) n_{-},
$$

where $q_{i, j}(h)=h-i t-j+N, c_{1} \in C$. Since $f^{N} e^{N} \equiv c_{2} h(h+1) \cdots(h+$ $n-1) \bmod U(g) n_{-}$, for some $c_{2} \in C$, we conclude that polynomial $p_{2}$ from lemma 3.4.4 is proportional to

$$
\prod_{i=0}^{l} \prod_{j=1}^{N}(h-i t-j+N) .
$$

Now, proposition follows from lemma 3.4.4.

We can obtain the main theorem:

Theorem 3.5.3. The set $\{L(k, r \omega): r \in S\}$ provides a complete list of irreducible $L(k, 0)$-modules from the category $\mathcal{O}$. Moreover, the irreducible $L(k, 0)$-modules from the category $\mathcal{O}$ are exactly irreducible highest weight representations with admissible highest weights.

Proof. Proposition 3.5.2 and theorem 2.2.1 imply that $L(k, r \omega)$, for $r \in$ $S$, are all irreducible $L(k, 0)$-modules from the category $\mathcal{O}$. The second statement follows from lemma 3.1.3.

Theorem 3.5.4. Let $M$ be a $L(k, 0)$-module from the category $\mathcal{O}$. Then $M$ is a completely reducible $L(k, 0)$-module. 
Proof. Let $M$ be a $L(k, 0)$-module from the category $\mathcal{O}$ and let $N$ be an irreducible subquotients of $M$. Then $N$ is an irreducible $L(k, 0)$-module. From Theorem 3.5.3, it follows that $N$ is an irreducible highest weight module with admissible highest weight. Now Theorem 3.1.5 implies that $M$ is a completely reducible $\hat{g}$-module and so a completely reducible $L(k, 0)$ module.

Remark 3.5.5. A vertex operator algebra is by definition rational if it has only finitely many irreducible modules and if every finitely generated module is a direct sum of irreducible ones. We have showed that VOA $L(k, 0)$ has finitely many irreducible modules in the category $\mathcal{O}$ and every module from the category $\mathcal{O}$ is completely reducible. By using these arguments we say that the vertex operator algebra $L(k, 0)$, for $k \in Q$ admissible, is rational in the category $\mathcal{O}$.

Remark 3.5.6. In $[\mathrm{A}]$ some modular invariant representations for $C_{\ell}^{(1)}$ were considered, and it was proved that the VOA $L\left(n-\frac{3}{2}, 0\right), n \in N$, is rational in the category $\mathcal{O}$.

We have

Conjecture 3.5.7. Let $g$ be any simple finite-dimensional Lie algebra and $L(k, 0)$ the associated vertex operator algebra such that the highest weight of $L(k, 0)$ is admissible. Then $L(k, 0)$ is rational in the category $\mathcal{O}$.

\section{Irreducible modules for $L(k, 0)$ in the category of weight modules}

Let $M$ be any irreducible $L(k, 0)$-module. From [FHL] we have that the countergradient $L(k, 0)$-module $M^{*}$ is also irreducible. Moreover, $M^{* *}$ and $M$ are isomorphic $L(k, 0)$-modules. One can easily see that for $M=$ $L(k, \lambda), M_{0}^{*}$ is isomorphic to $V(\lambda)^{*}$. We have

Proposition 4.0.8. If $r \in S$, then $V(r \omega)^{*}$ are all irreducible lowest weight $A(L(k, 0))$-modules.

Set $E_{r, \mu}=t^{\mu} C\left[t, t^{-1}\right]$ where $r, \mu \in C$ and $E_{i}=t^{\mu+i}$. Define a $U(g)$ action on $E_{r, \mu}$ by the following formulas:

$$
e . E_{i}=-(\mu+i) E_{i-1}, \quad h . E_{i}=(-2 \mu-2 i+r), \quad f . E_{i}=(\mu+i-r) E_{i+1} .
$$

We find all pairs $(r, \mu)$, such that $E_{r, \mu}$ is an irreducible $A(L(k, 0))$-module.

Theorem 4.0.9. Set $T=\left\{(r, \mu): r \in S-Z_{+}, \mu \notin Z, r-\mu \notin Z\right\}$. Then $E_{r, \mu}$ is an irreducible $A(L(k, 0))$-module if and only if $(r, \mu) \in T$. 
Proof. First, we notice that $E_{r, \mu}$ is an irreducible $U(g)$-module iff $\mu \notin Z$ and $r-\mu \notin Z$.

By using (11) we have

$$
Q \cdot E_{i}=\left(p_{0}(r)+p_{1}(r)(i+\mu)+\cdots+p_{N}(r)(i+\mu)^{N}\right) E_{i-N},
$$

for some polynomials $p_{0}, p_{1} \cdots p_{N}$ and $\mu \in C$.

Step 1. Let $E_{r, \mu}$ be an irreducible $A(L(k, 0))$-module, then $r \in S-Z_{+}$.

From Proposition 3.4.2, it follows that $Q . E_{i}=0$ for all $i \in Z$. From this fact and from (12), we have that

$$
p_{0}(r)=p_{1}(r)=\cdots=p_{N}(r)=0 .
$$

If $\mu=0$, we have that $C[t]$ is a submodule of $C\left[t, t^{-1}\right]$ isomorphic to $M(r \omega)$. From (12) and Proposition 3.4.2, it follows that $M(r \omega)$ is $A(L(k, 0))$ module. Then Theorem 3.5.3 implies that $r \in S-Z_{+}$(in this case $V(r \omega)=M(r \omega))$.

Step 2. If $(r, \mu) \in T$ then $E_{r, \mu}$ is the irreducible $A(L(k, 0))$-module.

Since $r \in S-Z_{+}$, we have that $M(r \omega)=V(r \omega) \cong C[t]$ and $Q . C[t]=0$. By using (11) for $\mu=0$, we conclude that $p_{0}(r)=p_{1}(r)=\cdots=p_{N}(r)=0$. This fact implies that

$$
Q . E_{i}=0 \quad \text { for all } \quad i \in Z \text {, }
$$

and we obtain that for $(r, \mu) \in T, E_{r, \mu}$ is an $A(L(k, 0))$-module.

Recall that a $U(g)$-module $U$ is called a weight module if $h$ acts semisimply on $U$ and all weight subspaces are finite-dimensional. We know that the irreducible weight modules are highest weight, lowest weight and modules $E_{r, \mu}$ defined by (11).

We have obtained

Corollary 4.0.10. Let $U$ be an irreducible $A(L(k, 0))$-weight module, then $U$ is one of the following modules:

(1) $V(r \omega), r \in S$,

(2) $V(r \omega)^{*}, r \in S$ or

(3) $E_{r, \mu},(r, \mu) \in T$.

Theorem 4.0.11. Let $M$ be an irreducible $L(k, 0)$-module such that $M_{0}$ is a weight module. Then $M$ is one of the following modules:

(1) $L(k, V(r \omega)), r \in S$,

(2) $L\left(k, V(r \omega)^{*}\right), r \in S$ or

(3) $L\left(k, E_{r, \mu}\right),(r, \mu) \in T$. 


\section{Connection with the geometrical approach}

Let $E$ be any finite subset of $C P^{1}$ and let $g(E)$ denote the Lie algebra of meromorphic functions on $C P^{1}$ holomorphic outside $E$ with values in $g$. For every $z \in C P^{1}$ and $\lambda \in h^{*}$ we can define an irreducible highest weight $g(z)$-module $L(k, \lambda, z)$ attached to $z$ (for definition see [FM]).

Let $z_{1}, z_{2}, z_{3}$ be three different points on $C P^{1}$ and $\lambda_{1}, \lambda_{2}, \lambda_{3} \in h^{*}$. Consider the $g\left(z_{1}, z_{2}, z_{3}\right)$-module $L\left(k, \lambda_{1}, z_{1}\right) \otimes L\left(k, \lambda_{2}, z_{2}\right) \otimes L\left(k, \lambda_{3}, z_{3}\right)$ and the space of coinvariants

$$
H^{\circ}\left(g\left(z_{1}, z_{2}, z_{3}\right), L\left(k, \lambda_{1}, z_{1}\right) \otimes L\left(k, \lambda_{2}, z_{2}\right) \otimes L\left(k, \lambda_{3}, z_{3}\right)\right) .
$$

In a previous section, we showed that the irreducible $L(k, 0)$-modules (in the category $\mathcal{O}$ ) are exactly $L(k, r \omega), r \in S$. Those modules were considered in $[\mathrm{FM}]$. They calculated the dimension of the space

$$
H^{\circ}\left(g(0,1, \infty), L\left(k, r_{1} \omega, 0\right) \otimes L\left(k, r_{2} \omega, 1\right) \otimes L\left(k, r_{3} \omega, \infty\right)\right),
$$

(known as conformal block), for all triples $r_{1}, r_{2}, r_{3} \in S$ and obtained the 'fusion algebra'.

When $r_{3}=0$, their result implies that

$$
\begin{aligned}
\operatorname{dim} H^{\circ}\left(g(0,1, \infty), L\left(k, r_{1} \omega, 0\right) \otimes L\left(k, r_{2} \omega, 1\right)\right. & \otimes L(k, 0, \infty)) \\
& = \begin{cases}1 & \text { if } r_{1}=r_{2} \\
0 & \text { otherwise }\end{cases}
\end{aligned}
$$

We have the following characterisation of $L(k, 0)$-modules:

Theorem 5.0.12. $L(k, \lambda)$ is a $L(k, 0)$-module if and only if

$$
\operatorname{dim} H^{\circ}(g(0,1, \infty), L(k, \lambda, 0) \otimes L(k, \lambda, 1) \otimes L(k, 0, \infty))=1 .
$$

As in [FHL], for three modules, we can define fusion rules (dimension of the space of intertwining operators). From the previous theorem, it follows that when one of the modules is $L(k, 0)$, then the fusion rules and the dimension of the corresponding conformal block are equal. It seems that this is true for any three modules.

\section{References}

[A] D. Adamović, Some rational vertex algebras, Glasnik Matematički 2 (1994), 25-41, q-alg/9502015.

[AY] H. Awata and Y. Yamada, Fusion rules for the fractional level sl $(2)^{\sim}$ algebra, KEK-TH-316 KEK Preprint 91-209, 1992.

[Dix] J. Dixmier, Algebres envelopantes, Gauthier-Villard, 1978.

[DL] C. Dong and J. Lepowsky, Generalized vertex algebras and relative vertex operators, Progress in math. 112, Birkhauser, Boston, 1993. 
[F] D. B. Fuchs Projekcija osobije vektora modula Verma algebre $A_{1}^{1}$, Funkc. Anal. I Ego. Priloženja 2 (1989), 82-86.

[FHL] I. Frenkel, Y.-Z.Huang and J. Lepowsky, An axiomatic approach to Vertex operator algebras and modules, Memoirs of the AMS 494, 1994.

[FLM] I. Frenkel, J. Lepowsky and A. Meurman, Vertex operator algebras and the monster, Pure and appl. math. 134, Academic Press, Boston, 1988.

[FM] B. Feigin and F. Malikov, Fusion algebra at a rational level and cohomology of nilpotent subalgebras of sl(2) $\sim$, Letters in Math. Phsics 31 (1994), 315-325.

[FZ] I. Frenkel and Y.-C.Zhu, Vertex operator algebras associated to representations of affine and Virasoro algebras, Duke Math. J. 66 (1992), 123-168.

[K] V. Kac, Infinite dimensional Lie algebras, Cambridge Univ. Press, 1990.

$[\mathrm{KW}]$ V. Kac and M. Wakimoto, Modular invariant representations of infinite dimensional Lie algebras and superalgebras, Proc. Natl. Acad. Sci. 85 (1988), $4956-4960$

[KWn] V. Kac and W. Wang, Vertex operator superalgebras and their representations, preprint.

[MFF] F. G. Malikov, B. L. Feigin and D. B. Fuchs, Osobije vektori v moduljah Verma nad algebrama Kaca-Mudi, Funkc. Anal. I Ego priloženja 20 (1986), 25-37.

[MP] A. Meurman and M. Primc, Annihilating fields of $\operatorname{sl}(2, C) \sim$ and combinatorial identities, preprint.

[W] W. Wang, Rationality of Virasoro vertex operator algebra, Duke Math. J. IMRN 71 (1993), 197-211.

[Z] Y.-C. Zhu, Vertex operator algebras, elliptic function and modular forms, $\mathrm{PhD}$ Thesis, Yale University, 1990.

Department of Mathematics, University of Zagreb, BijeničKa 30, 41000 ZAGREB, CROATIA

E-mail address: adamovic@cromath.math.hr

Department of Mathematics, University of Zagreb, BijeničKa 30, 41000 ZAGreb, Croatia

E-mail address: milas@cromath.math.hr 